\title{
Computational Study on Wingtip Vertical Fluid Injection for Induced Drag Reduction
}

\author{
Hariprasad Thimmegowda ${ }^{1}$, Yadu Krishnan $S^{1}$, Gisa G S ${ }^{1}$ \\ ${ }^{1}$ Alliance University \\ Anekal, Bangalore, India \\ hariprasad.thimmegowda@alliance.edu.in; yadukrishnan.s@alliance.edu.in \\ gisa.gs@alliance.edu.in
}

\begin{abstract}
The present study examines the numerical analysis of the effects of wingtip vertical fluid injection on the performance of a 3D wing. A wing configuration was chosen as a baseline configuration and a slot was created at the wingtip to inject the fluid vertically. The investigation was performed at various incidence angles along with a range of fluid injection speeds. The reduction in wingtip vortices, due to the presence of the vertical fluid injection at the wingtip, was observed. In turn, an improvement in pressure distribution around the wingtip was apparent. With an increase in injection velocity, the drag values were seen to decrease. However, for all injection flowrates, the lift-to-drag ratio increases. This alternate control method can be used to improve the aerodynamic efficiency of the wing by reducing the induced drag.
\end{abstract}

Keywords: Induced drag, Winglets, Fluid injection, CFD.

\section{Introduction}

In the present time, the major challenge in the aviation industry is reducing fuel consumption as fuel costs are constantly increasing. To significantly limit fuel utilization, sufficient exploration has been done in past and at present far and wide.

The primary task of an aircraft designer is to improve aerodynamic efficiency. The profile of an airfoil and lifting device influences the lift generation, which in turn, varies with changes in the angle of attack. The maximum lift-to-drag ratio occurs, at a particular value of lift coefficient and the corresponding angle of attack. In steady level flight, the total drag is minimal, consequently, the lift to drag ratio is maximum. Nonetheless, if the approach veers off from the one making the greatest liftto-drag ratio, then the lift-drag ratio reduces, in turn, increasing the total drag for a given lift of an aircraft. Based on Bernoulli's principle, the pressure on the upper surface is smaller and the pressure on the lower surface is higher on the wing. This generates the pressure difference between the two surfaces of the wing. However, the pressure difference tries to equalize itself at the wingtips, producing trailing vortices that generate induced drag.

The lift-induced drag is one of the key contributors to the overall drag and can be minimized by weakening the trailing vortices. The author [1], in 1970, studied the winglet concept patented by F W Lancaster in the late 1800s. The investigation has shown that winglets reduced the induced drag by about 20 percent and increased the lift-drag ratio approximately by 9 percent. Therefore, by implementing winglets on wingtips, the wind tunnel results predicted improved aerodynamic and cruising efficiencies. To enhance aerodynamic performance, research on many kinds of winglet designs has been explored ever since. Based on a biomimetic imitation Spiroid-tipped wing [2,3] that bends upward by 360 degrees to form the large rigid ribbon, and multi-element winglets [4-7], mimicking birds, with various cant angles and element configuration was developed. To improve wing performance, qualitative and quantitative optimization analyses on blended winglets and split blended wings have been investigated [8-12]. The author [13] used winglets on a saucer-shaped aircraft that is a result of blending fuselage with the wing. When a sweep-back fin-shaped winglet is added, the ratio of lift to drag increases by 75 percent, and the coefficient of lift also increases by adding the winglet. The study also revealed, from flight tests, that the load capability and lateral stability are improved by using the winglets. All these studies suggest improving aerodynamic efficiency.

Winglets, however, are a structural modification and thus form a permanent attachment present for the entire flight envelope. Winglets are efficient in reducing the induced drag, though, there is a possibility of an increase in drag due to the skin friction with the addition of winglets. Handling qualities can be affected by retrofitting the winglets [14]. The wing twist alters because of aeroelastic effects and hence affects the performance of the aircraft. The authors [15-17] have examined 
the impact of the winglet on long-range aircraft. This research indicates that winglets create flutter issues and add extra mass at the wingtips which in turn create rolling inertia along with the bending load.

The aircraft cruise conditions include a lower angle of attack in which the fraction of induced drag to the total drag may not be significant. However, its contribution is considerable during landing and take-off due to the larger angle of attack. Thus, it is prudent to utilize a winglet when the need arises. This cannot be accomplished with a permanent winglet. The authors [18], investigated the effect of fluidic winglets on the aerodynamic efficiency of the wing on demand. In the reference [18] fluid is injected into flow in a horizontal direction at the wingtip.

An on-demand solution, a fluidic winglet is explored in this paper as a substitute for a permanent winglet. At the tip of the wing, the fluid will be pumped in the vertical direction. When the fluid is injected vertically, the flow at the wingtip will be altered. To understand the impact of the introduction of fluid on-demand at the wingtip in the vertical direction, a numerical analysis has been carried out.

\section{Numerical Methodology}

The baseline wing consists of a NACA 0012 aerofoil profile. The wing is designed to have a chord of $100 \mathrm{~mm}$ and a span of $300 \mathrm{~mm}$ keeping the Aspect ratio 3. The aerofoil section of the wing considered is shown in figure 1.

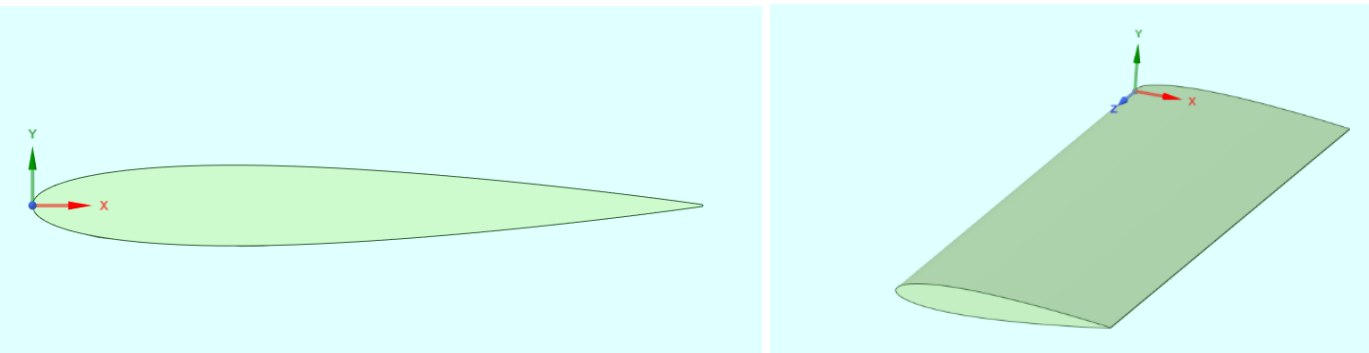

Fig. 1: Aerofoil section of the wing - NACA 0012

Fig. 2: 3D wing model

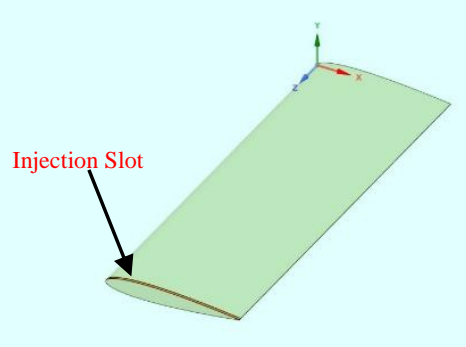

Fig. 3: Wing with injection slot

The CFD domain for the baseline wing, with and without fluid injection, is identical for the analysis. The slot dimension is $2 \mathrm{~mm}$ at the tip on the upper surface of the wing. The injection slot is created from the leading edge to the trailing edge as shown in figure 3.

Since the gradient in flow properties like velocity and pressure are high near the wing, a fine mesh is generated around the wing surface using the sphere of influence method, and the flow properties gradient is so small far away from the wing surface, a coarse mesh is used in this area. Inflation layers have been given to capture gradient information at the fluidsurface boundaries (figure 4). 

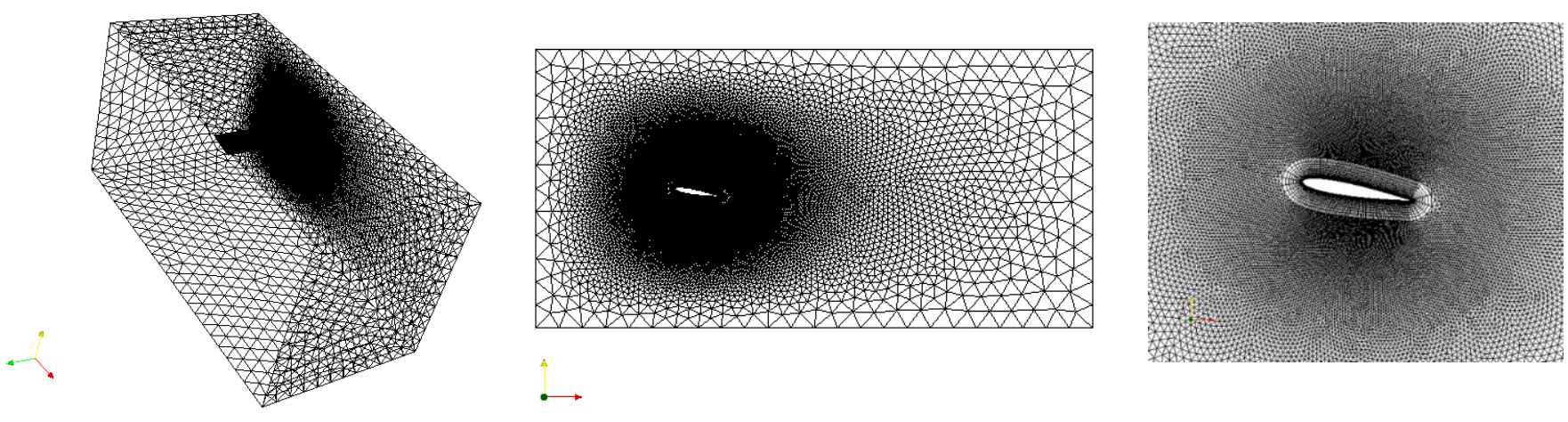

Fig. 4: Computational domain and meshing

\subsection{The Boundary Conditions}

The wall boundary condition is applied to the surrounding walls and wing. Velocity inlet and outflow are used for inlet and exit boundaries. The inlet velocity is $20 \mathrm{~m} / \mathrm{s}$ and ambient pressure is $1 \mathrm{~atm}$ at the inlet. A velocity inlet is given for the injection slot as shown in figure 5 .
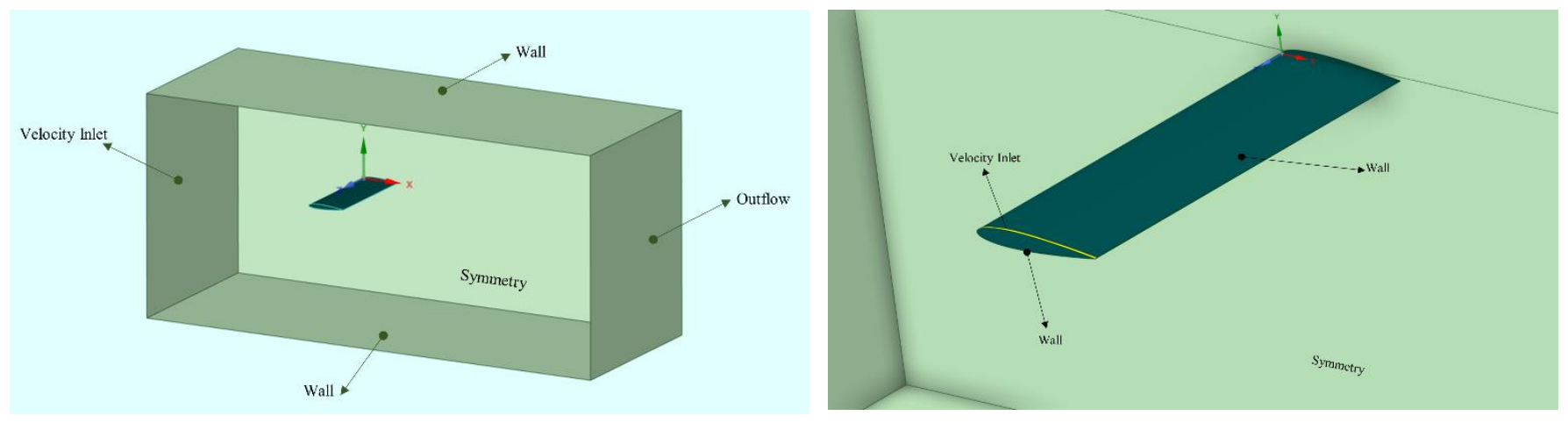

Fig. 5: Boundary conditions

\section{Results and Discussions}

In the present case, the baseline wing at $10^{\circ}$ angle of attack with an aspect ratio of 3 , is considered for validation. The drag and lift values obtained from the numerical simulations of mentioned configuration are validated with [19]. Table 1 shows the comparison. The force values are obtained from the numerical analysis and the coefficient of forces is calculated using the reference values.

Table- 1: Validation of CFD results with Experimental result

\begin{tabular}{|c|c|c|}
\hline Coefficients & Experiment result [19] & CFD result \\
\hline $\mathrm{C}_{\mathrm{L}}$ & 0.6 & 0.654 \\
\hline $\mathrm{C}_{\mathrm{D}}$ & 0.12 & 0.1107 \\
\hline
\end{tabular}

\subsection{Baseline}

The pressure distribution for the baseline wing at an angle of attack, $10^{\circ}$ is shown in figure 6 . The pressure distribution shows that it drops rapidly near the leading edge before regaining its previous values in the downstream direction. This depicts the flow accelerating along the leading edge and then decelerating as it flows downstream (figure 7). 


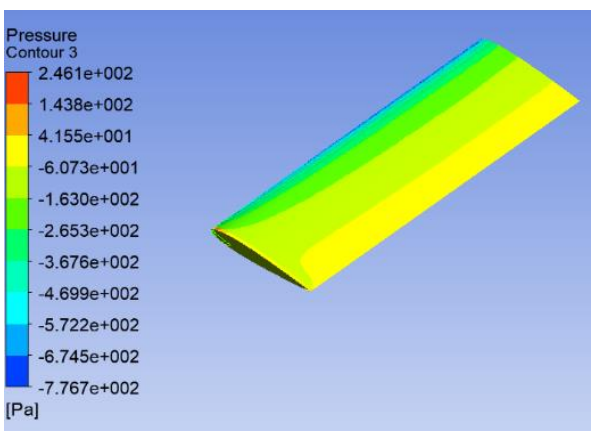

a)

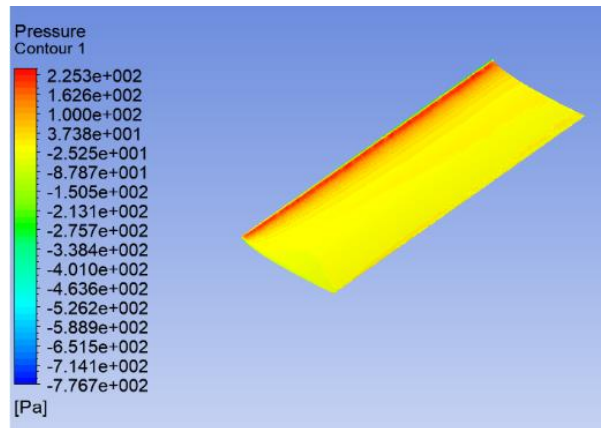

b)

Fig. 6: Pressure distribution on (a) top (b)bottom surface of baseline wing at $\alpha 10^{\circ}$

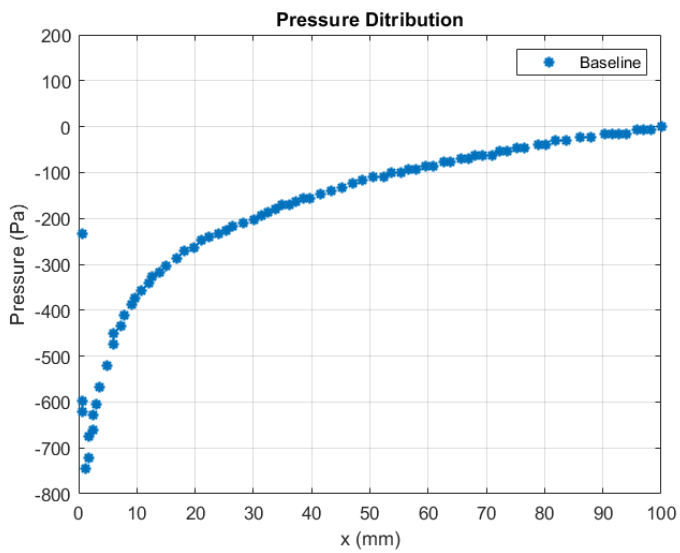

Fig. 7: Pressure distribution on the top surface of baseline wing at $\alpha=10^{\circ}$

A small vortex is seen in the flow near the tip of the wing, which increases in size as it travels downstream. The flow at downstream is seen to change the direction along the wingspan towards the wing root, due to the action of the wingtip vortex. This is seen in the comparison of pressure distribution at various span locations which is shown in figure 8 .

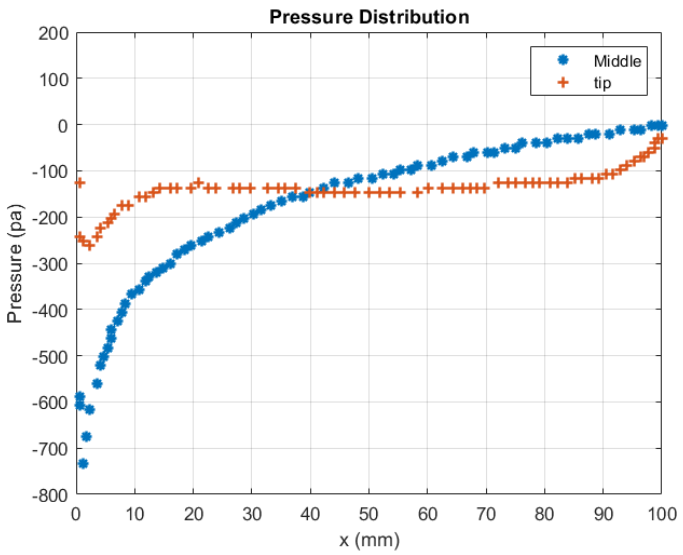

Fig. 8: Comparison Pressure distribution on the top surface at various span location of baseline wing at $\alpha=10^{\circ}$

\subsection{With fluid Injection}

The top surface pressure distribution for the wing with injection at an angle of attack of $10^{\circ}$ is shown in figure 9(a). Near the tip, the pressure distribution seems to be altered by the presence of the injection. A minor variation could be observed in the pressure distribution on the bottom surface of the wing as shown in figure $9(\mathrm{~b})$. 
The comparison of pressure at $\mathrm{z}=150 \mathrm{~mm}$ and tip, with and without injection, at an angle of attack of $10^{\circ}$ is shown in Figures 10 and 11 respectively. In figure 10, it is observed that the pressure distribution along the chord on the baseline wing is comparable with the wing having an injection velocity of $30 \mathrm{~m} / \mathrm{s}$. However, at the tip of both wings, a significant variation in the pressure distribution is noticed as depicted in figure 11. This indicates the effect of injection at the tip. Due to this effect, the vortex strength is reduced at the tip, nevertheless, the flow is undisturbed at other locations on the span. The streamlines near the tip of both the wing configurations are shown in figures 12 (a) and (b) respectively. The difference in streamlines indicates that the injection is altering the streamlines which helps to achieve a reduction in induced drag.

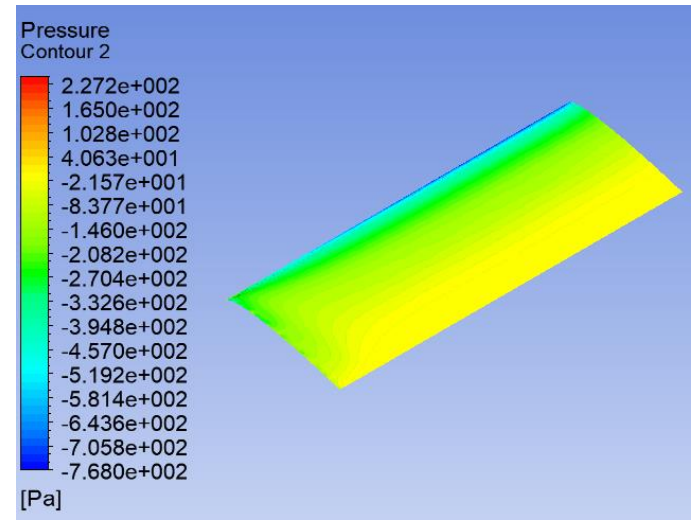

a)

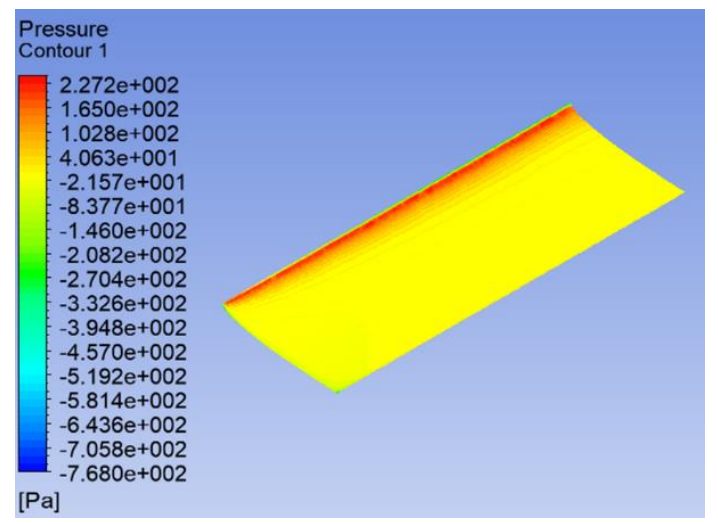

b)

Fig. 9: Pressure distribution on (a) top surface and (b) bottom surface of the wing with $30 \mathrm{~m} / \mathrm{s}$ injection velocity at $\alpha 10^{\circ}$

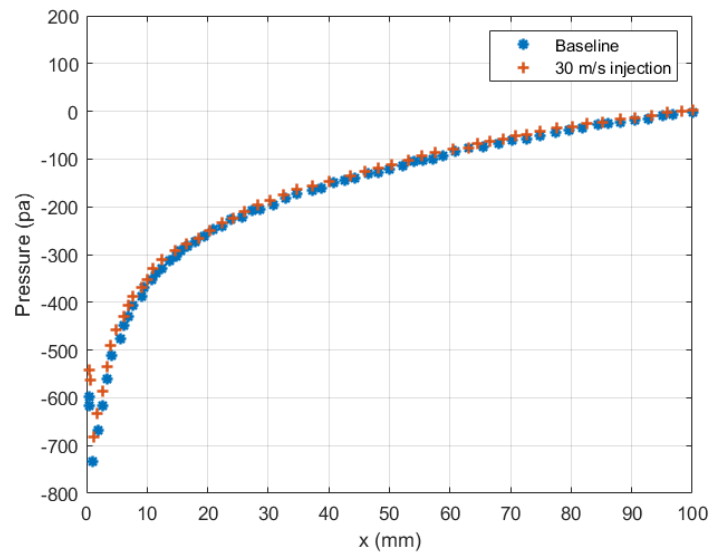

Fig. 10: Effect of injection on Pressure distribution at $\mathrm{z}=150 \mathrm{~mm}$ at $\alpha=10^{\circ}$

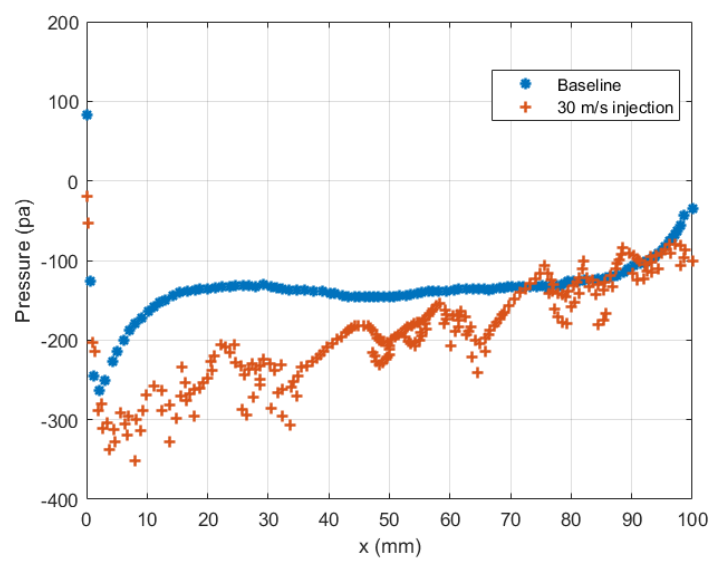

Fig. 11: Effect of injection on Pressure distribution at the tip at $\alpha 10^{\circ}$ 


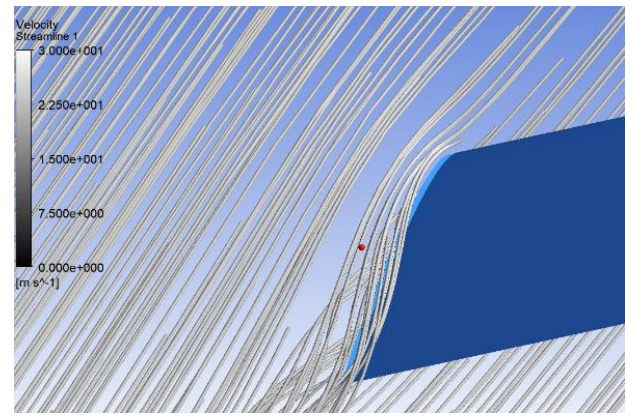

a)

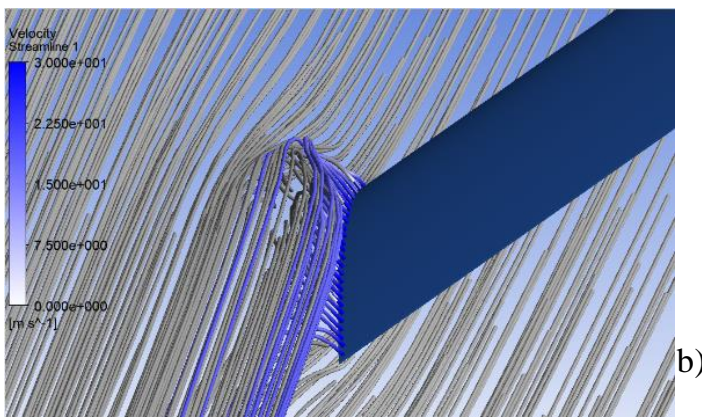

Fig. 12: Streamlines near the tip of the wing a) baseline b) with injection at $30 \mathrm{~m} / \mathrm{s}$

\subsection{Variation of aerodynamic coefficients}

The variation of coefficient of lift, coefficient of drag, and their ratio for all the configurations are shown from figures 13 to 15 . The lift remains constant and the drag values decrease with the increase in injection velocity for all the angles of attack. The key thing to note here is that the drag can be decreased by almost 20 percent by fluid injection. Since the coefficient of lift remains constant and the L/D ratio slightly increases with an increase in fluid injection velocities as there is a decrease in coefficient of drag.

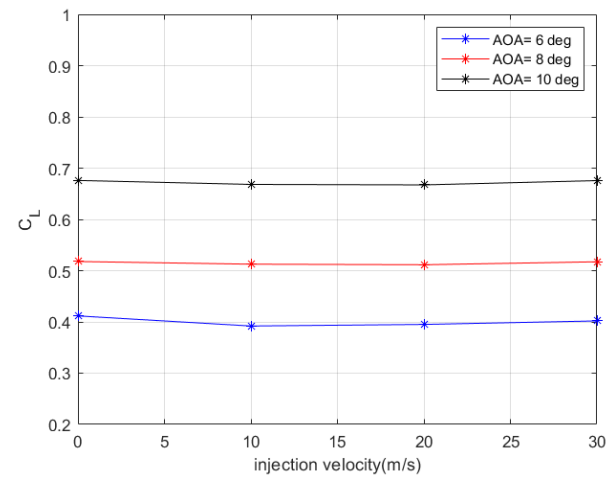

Fig. 13: Variation of lift with injection velocities

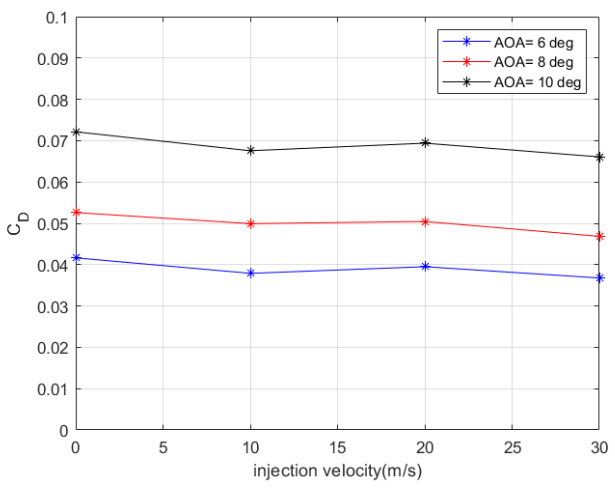

Fig. 14: Variation of drag with injection velocities

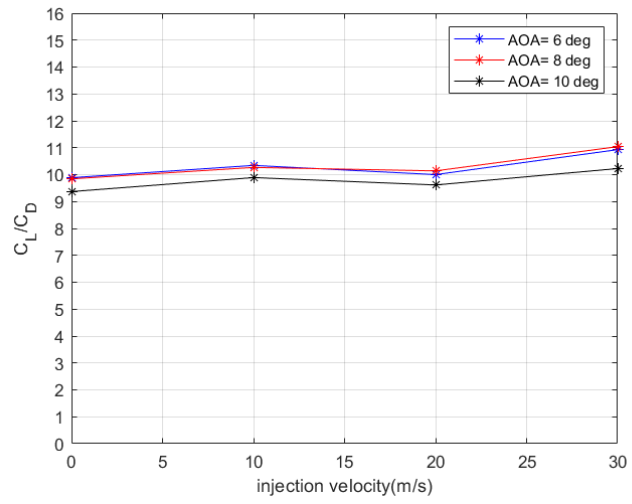

Fig. 15: Variation of L/D ratio with an injection velocity

\section{Conclusions}

The effect of fluid injection, where a slot is used at the tip of the wing to inject the fluid vertically at the wingtip, on the efficiency of a 3D wing at velocities of $10 \mathrm{~m} / \mathrm{s}, 20 \mathrm{~m} / \mathrm{s}$, and $30 \mathrm{~m} / \mathrm{s}$ was analyzed using the numerical approach at angles of attack $6^{\circ}, 8^{\circ}$, and $10^{\circ}$. It is observed for angles of attack $6^{\circ}, 8^{\circ}$, and $10^{\circ}$, the $C_{L}$ remains constant for every injection velocity. Comparing with the previous numerical investigation on horizontal injection system, vertical injection system has better $\mathrm{C}_{\mathrm{L}}$ 
characteristics. In turn, better L/D ratios. However, the drag coefficient is high for vertical fluid injection systems in comparison with horizontal injection systems at lower angles of attack. Therefore, the authors conclude that at lower angles of attack horizontal injection system can be used while at higher angles of attack vertical injection system can be used. Careful usage of fluid injection can be a good replacement for conventional wingtip devices.

\section{References}

[1] Whitcomb, Richard T. "A Design Approach and Selected Wind-Tunnel Results at High Subsonic Speeds for Wing-Tip Mounted Winglets,". Washington: National Aeronautics and Space Administration, 1976.

[2] Gratzer LB, inventor; Gratzer Louis B, assignee. "Spiroid-tipped wing,". United States patent US 5,102,068. Apr 7,1992 .

[3] J. Guerrero, D. Maestro and A. Bottaro, "Biomimetic spiroid winglets for lift and drag control," Comptes Rendus Mécanique, vol. 340, no. 1-2, pp. 67-80, 2012. Available: 10.1016/j.crme.2011.11.007.

[4] Hossain A, Rahman A, Hossen J, Iqbal P, Shaari N, Sivaraj GK. "Drag reduction in a wing model using a bird feather like winglet," Jordan Journal of Mechanical and Industrial Engineering, June 1, 2011,5(3).

[5] Reddy SR, Sobieczky H, Dulikravic GS, Abdoli A. "Multi-element winglets: multi-objective optimization of aerodynamic shapes," Journal of Aircraft. July,2016, 53(4):992-1000.

[6] Giridharan V, Kumar KM, Elumalai N, Sundararaj M. "Computational study of effectiveness of winglet at subsonic speed,”. In AIP Conference Proceedings Sep 28, 2020 (Vol. 2271, No. 1, p. 030028). AIP Publishing LLC.

[7] Smith M, Komerath N, Ames R, Wong O, Pearson J. "Performance analysis of a wing with multiple winglets," In 19th AIAA Applied Aerodynamics Conference 2001 (p. 2407).

[8] Gratzer LB, inventor; Gratzer Louis B, "Blended winglet," United States patent US 5,348,253, Sep 20,1994

[9] Belferhat, S., S. M. A. Meftah, T. Yahiaoui, and B. Imine. "Aerodynamic Optimization of a Winglet Design," In EPJ Web of Conferences, vol. 45, p. 01010. EDP Sciences, 2013.

[10] Khosravi, Shahriar, and David W. Zingg. "A numerical optimization study on winglets," In 15th AIAA/ISSMO multidisciplinary analysis and optimization conference, p. 2173. 2014.

[11] Gratzer, Louis B. "Split blended winglet," U.S. Patent 8,944,386, issued February 3, 2015.

[12] Reddy, Sohail R., George S. Dulikravich, Abas Abdoli, and Helmut Sobieczky. "Multi-winglets: Multi-objective optimization of aerodynamic shapes," In 53rd AIAA Aerospace Sciences Meeting, p. 1489. 2015.

[13] YU JL, WANG LL, Ge G. "Using wing tip devices to improve performance of saucer-shaped aircraft," Chinese Journal of Aeronautics, Nov 1, 2006,19(4):309-14.

[14] van Dam C P, Holmes BJ, Pitts C. "Effect of winglets on performance and handling qualities of general aviation aircraft," Journal of Aircraft, July 18, 1981 (7):587-91.

[15] Delavenne M, Barriety B, Vetrano F, Ferrand V, Salaun M. "Assessment of the efficiency of an active winglet concept for a long-range aircraft," CEAS Aeronautical Journal. Dec 11, 2020 (4):971-90.

[16] Hantrais-Gervois JL, Rapin M. “Aerodynamic and structural behaviour of a wing equipped with a winglet at cruise," In 44th AIAA Aerospace Sciences Meeting and Exhibit, Jan ,2006, (p. 1489).

[17] Odaguil, Felipe Issamu Kitadani, A. Pequeno Antunes, Pedro Paglione, and João Luiz F. Azevedo. "Performance assessment of a morphing wingtip device," In International congress of mechanical engineering, Ribeirao Preto, Br. 2013.

[18] Rajesh A, Badri RA, Prasad MS. "Numerical Analysis on the Effect of Fluidic on Demand Winglet on the Aerodynamic Performance of the Wing," Journal of Aeronautics and Aerospace Engineering, 2017,6(3):198-203.

[19] Ananda GK, Sukumar PP, Selig MS. "Measured aerodynamic characteristics of wings at low Reynolds numbers," Aerospace Science and Technology, April 1,2015 42:392-406.

[20] Abbott IH, Von Doenhoff AE. "Theory of wing sections: including a summary of airfoil data," Courier Corporation, Apr 26, 2012 